\title{
SPACES OF MEASURES ON COMPLETELY REGULAR SPACES
}

\author{
C. CONSTANTINESCU
}

Let $X$ be a regular topological space. If $\left(\mu_{n}\right)_{n \in N}$ is a sequence of Radon (i.e., inner regular by compact) measures on $X$ such that $\left(\mu_{n}(T)\right)_{n \in N}$ converges for every regular open set $T$ of $X$ (i.e., for which $\stackrel{\circ}{T}=T)$, then $\left(\mu_{n}(A)\right)_{n \in N}$ converges for every Borel set $A$ of $X$. This result was proved by P. Gänssler ([4] Theorem 3.1) for real measures and by S. S. Khurana ([7] Theorem 4) for group valued measures. It will be shown in this paper (Theorem 3) that, if $X$ is completely regular, this result can be improved by assuming only that $\left(\mu_{n}(T)\right)_{n \in N}$ converges for those regular open sets $T$ of $X$ for which there exists a continuous real function $f$ on $X$ such that

$$
\{f>0\} \subset T \subset \overline{\{f>0\}}
$$

(or equivalently $T=\overline{\{f>0\}}$ ); we denote the set of these sets $T$ by $\mathfrak{I}$. If the vector lattice of continuous real functions on $X$ is order $\sigma$-complete, then $\mathfrak{I}$ is exactly the set of closed open sets of $X$ and so the above formulation contains the corresponding result of $Z$. Semadeni ([8] Theorem (i) $\Rightarrow($ iv)). Let $\mathscr{C}$ be the vector space of continuous bounded real functions on $X$ endowed with the strict topology and $E$ be a quasicomplete $G$-space ([2] Definition 5.9.11). We show (Theorem 12) that a continuous linear map $u: \mathscr{C} \rightarrow E$ is boundedly weakly compact (or equivalently possesses an integral representation) if and only if the sets of $\mathfrak{I}$ are sent into $E$ by the biadjoint map of $u$. The special case of $E$ equal to the vector space of continuous real functions on a metrizable topological space endowed with the topology of compact convergence is discussed in greater detail (Theorems 15 and 16).

We use the notations and the terminology of [1] and [2]. The expression locally convex space will mean Hausdorff real locally convex space. For every locally convex space $E$ we denote its dual and bidual by $E^{\prime}$ and $E^{\prime \prime}$, respectively, and identify $E$ with a subspace of $E^{\prime \prime}$ via the evaluation map

$$
E \rightarrow E^{\prime \prime}, \quad x \mapsto\langle x, \cdot\rangle .
$$

For every continuous linear map $u$ of locally convex spaces, $u^{\prime}$ and $u^{\prime \prime}$ will denote the adjoint and the biadjoint of $u$, respectively. $\boldsymbol{N}, \boldsymbol{Q}, \boldsymbol{R}$ denote the sets of natural numbers, rational numbers, and real numbers respectively.

Throughout this paper we denote by $E$ a locally convex space, by $Y$ a completely regular space, by $X$ a subspace of $Y$, by $\mathscr{C}$ the vector space of continuous bounded 
real functions on $X$ endowed with the strict topology, by $\mathfrak{R}$ the set of compact sets of $X$, by $\Re$ the $\sigma$-ring of Borel sets of $X$, and by $\mathscr{M}$ the band $\mathscr{M}(\mathfrak{R}, \boldsymbol{R} ; \mathfrak{R})$ of $\mathscr{M}(\mathfrak{R}, \boldsymbol{R})([2] \text { Proposition 5.6.3) })^{1}$. For every subset $A$ of $X$ we denote its closure and its interior in $X$ by $\bar{A}$ and by $\AA$, respectively, and we set ${ }^{2)}$

$$
\mathfrak{I}:=\left\{\frac{0}{V \cap X} \mid V \text { exact open set of } Y\right\} \text {. }
$$

For every set $T$ of $\mathfrak{I}$ there exists an exact open set $V$ of $X$ such that $T=\stackrel{\circ}{V}$; hence $T$ is an open regular set of $X$. But it may happen that $\mathfrak{I}$ is strictly contained in the set

$$
\{\stackrel{\circ}{V} \mid V \text { exact open set of } X\},
$$

and this will make our results more general. This is the reason for the introduction of $Y$.

$Y$ will be called $\sigma$-Stonian if the vector lattice of continuous real functions on $Y$ is order $\sigma$-complete. This is equivalent to the assertion that the closure of every exact open set of $Y$ is open ([2] Lemma 5.9.15 a $\Leftrightarrow$ c). If $Y$ is $\sigma$-Stonian, then every set of $\mathfrak{I}$ is a closed open set of $X$.

Proposition 1. The set $\mathfrak{I}$ is a base of $X$ closed with respect to finite intersections such that $\overline{\bigcup_{\imath \in I} T_{\imath}} \in \mathfrak{T}$ for every countable family $\left(T_{\imath}\right)_{\imath \in I}$ in $\mathfrak{I}$.

Let $x \in X$ and let $U$ be a neighbourhood of $x$ in $X$. There exists a neighbourhood $V$ of $x$ in $Y$ such that $V \cap X \subset U$. Further, there exists a continuous real function $f$ on $Y$ equal to 0 at $x$ and equal to 2 on $Y \backslash V$. We set

$$
W:=\{f<1\}, \quad T:=\overline{W \cap X} .
$$

Then $x \in T \subset U$ and $T \in \mathfrak{I}$. Hence $\mathfrak{I}$ is a base of $X$.

Let $T^{\prime}, T^{\prime \prime} \in \mathfrak{I}$. There exist exact open sets $V^{\prime}, V^{\prime \prime}$ of $Y$ such that

$$
T^{\prime}=\overline{V^{\prime} \cap X}, \quad T^{\prime \prime}=\overline{V^{\prime \prime} \cap X} .
$$

We set $T:=T^{\prime} \cap T^{\prime \prime}, \quad V:=V^{\prime} \cap V^{\prime \prime}$. Then $V$ is an exact open set of $Y$ and

$$
\overline{V \cap X}=\overline{\left(V^{\prime} \cap X\right) \cap\left(V^{\prime \prime} \cap X\right)} \subset T .
$$

Let $U$ be a nonempty open set of $X$ contained in $T$. Since $T \subset \overline{V^{\prime}} \overline{\cap X}$, the set $U \cap V^{\prime} \cap X$ is nonempty. Since $T \subset \overline{V^{\prime \prime} \cap X}$, the set $\left(U \cap V^{\prime} \cap X\right) \cap\left(V^{\prime \prime} \cap X\right)$ is also

1) Let $F$ be a complete vector lattice; a band of $F$ is a vector subspace $G$ of $F$ such that:

$$
x \in F, y \in G,|x| \leqq|y| \Rightarrow x \in G, \quad x \in F, x=\underset{G \ni y \leqq x}{\bigvee} y \Rightarrow x \in G .
$$

2) An open set $V$ of $Y$ is called exact if there is a continuous real function $f$ on $Y$, such that

$$
V=\{x \in Y \mid f(x)>0\} .
$$


nonempty. Hence $T \subset \overline{V \cap X}$ and we get

$$
T=\overline{V \cap X} \in \mathfrak{I}
$$

This shows that $\mathfrak{I}$ is closed with respect to finite intersections.

Let $\left(T_{\imath}\right)_{\imath \in I}$ be a countable family in $\mathfrak{I}$. For every $\imath \in I$ there exists an exact open set $V_{\imath}$ of $Y$ such that $T_{\imath}=\overline{V_{\imath} \cap X}$. We set $V:=\bigcup_{\imath \in I} V_{\imath}$. Then $V$ is an exact open set of $Y$ and

$$
\begin{gathered}
V \cap X=\bigcup_{\imath \in I}\left(V_{l} \cap X\right) \subset \bigcup_{\imath \in I} T_{l} \subset \bigcup_{\imath \in I} \overline{V_{\imath} \cap X} \subset \overline{V \cap X}, \\
\overline{\bigcup_{\imath \in I} T_{\imath}}=\overline{V \cap X} \in \mathfrak{I} .
\end{gathered}
$$

Proposition 2. Let $K$ be a compact set of $X$ and $F$ be a closed set of $X$ such that $K \cap F=\emptyset$. Then there exist disjoint sets $T^{\prime}, T^{\prime \prime} \in \mathfrak{I}$ such that $K \subset T^{\prime}, F \subset T^{\prime \prime}$. If $X$ is normal and equal to $Y$, then we may take $K$ closed.

There exists a continuous real function $f$ on $Y$ such that $f=0$ on $K$ and $f=2$ on $F$. The sets $\{f<1\},\{f>1\}$ are exact open sets of $Y$ and so the sets

$$
T^{\prime}:=\overline{\{f<1\} \cap X}, \quad T^{\prime \prime}:=\overline{\{f>1\} \cap X}
$$

possess the required properties.

Theorem 3. The identity map

$$
\mathscr{M}(\mathfrak{R}, G ; \mathfrak{R})_{\mathfrak{T}} \rightarrow \mathscr{M}(\mathfrak{R}, G ; \Re)
$$

is uniformly $\Phi_{3}$-continuous for every Hausdorff topological additive group $G$. If $X$ is normal and equal to $Y$ then we may replace $\Re$ by the set of closed sets of $X$.

Let $\mathfrak{L}$ be the set of closed sets of $X$. We want to use Theorem 4.5 .13 of [2] in order to show that the identity map

$$
\mathscr{M}(\mathfrak{R}, G ; \mathfrak{R})_{\mathfrak{I}} \rightarrow \mathscr{M}(\mathfrak{R}, G ; \mathfrak{R})_{\mathfrak{L}}
$$

in uniformly $\Phi_{3}$-continuous. In fact, the hypotheses a), d), and e) of that theorem follow from Proposition 2 and the hypotheses b) and c) from Proposition 1. By [2] Proposition 4.5.6 the identity map

$$
\mathscr{M}(\mathfrak{R}, G ; \mathfrak{R})_{\mathfrak{I}} \rightarrow \mathscr{M}(\mathfrak{R}, G ; \mathfrak{R})
$$

is uniformly $\Phi_{4}$-continuous and so by [2] Corollary 1.8 .5 the identity map

$$
\mathscr{M}(\mathfrak{R}, G ; \mathfrak{R})_{\mathfrak{T}} \rightarrow \mathscr{M}(\mathfrak{R}, G ; \mathfrak{R})
$$

is uniformly $\Phi_{3}$-continuous. 
Remarks 1. The assertion and the proof still hold if we replace $\mathfrak{R}$ by a $\sigma$-ring of subsets of $X$ containing $\mathfrak{I}$ and $\mathfrak{R}$ by the set of compact sets (closed sets if $X$ is normal and equal to $Y$ ) of $X$ belonging to $\Re$. This remark also holds for Corollary 4 .

2. If $Y$ is $\sigma$-Stonian, then the sets of $\mathfrak{I}$ are closed open sets of $X$. Hence the above formulation has the advantage of unifying the corresponding results with open regular sets ([2] Corollary 4.5.15) and with closed open sets ([2] Corollary 4.5.17).

Corollary 4. If $E$ is quasicomplete, then $\int \xi d \mu \in E$ for every $(\xi, \mu) \in \mathscr{M}^{\pi} \times \mathscr{M}(E)$ and the identity map

is uniformly $\Phi_{3}$-continuous.

$$
\mathscr{M}(E)_{\mathfrak{I}} \rightarrow \mathscr{M}(E)_{\mathscr{M}^{\pi}}
$$

By [1] Theorem 4.2.11, $\int \xi d \mu \in E$ for every $(\xi, \mu) \in \mathscr{M}^{\pi} \times \mathscr{M}(E)$. By Theorem 3 the identity map

$$
\mathscr{M}(E)_{\mathfrak{I}} \rightarrow \mathscr{M}(E)
$$

is uniformly $\Phi_{3}$-continuous and the assertion follows from [2] Theorem 5.6.6.

Proposition 5. We have:

a)

b) the map

$$
\mathscr{C} \subset \bigcap_{\mu \in \mathscr{M}} \mathscr{L}^{1}(\mu) ;
$$

$$
\mu^{\prime}: \mathscr{C} \rightarrow \boldsymbol{R}, \quad f \mapsto \int f d \mu
$$

belongs to $\mathscr{C}^{\prime}$ for every $\mu \in \mathscr{M}$;

c) the map

$$
\left(\mathscr{M}, \mathscr{M}^{\pi}\right) \rightarrow \mathscr{C}^{\prime}, \quad \mu \mapsto \mu^{\prime}
$$

is an isomorphism of Banach spaces;

d) the map

$$
u^{\prime}: \mathscr{M} \rightarrow \boldsymbol{R}, \quad \mu \mapsto u\left(\mu^{\prime}\right)
$$

belongs to $\mathscr{M}^{\pi}$ for every $u \in \mathscr{C}^{\prime \prime}$;

e) the map

$$
\mathscr{C}^{\prime \prime} \rightarrow \mathscr{M}^{\pi}, \quad u \mapsto u^{\prime}
$$

is an isomorphism of vector spaces.

The assertions follow from [5] Theorem 4.6 and Theorem 2.4 (iii) and [1] Proposition 3.4.2 b).

Remark. We identify $\mathscr{M}$ with $\mathscr{C}^{\prime}$ and $\mathscr{M}^{\pi}$ with $\mathscr{C}^{\prime \prime}$ via the above isomorphisms.

Theorem 6. The identity map $\left(\mathscr{C}^{\prime}\right)_{\mathfrak{T}} \rightarrow\left(\mathscr{C}^{\prime}\right)_{\mathscr{C}^{\prime \prime}}$ is uniformly $\Phi_{3}$-continuous. If $Y$ is $\sigma$-Stonian, then the identity map $\left(\mathscr{C}^{\prime}\right)_{\mathscr{C}} \rightarrow\left(\mathscr{C}^{\prime}\right)_{\mathscr{C}^{\prime \prime}}$ is uniformly $\Phi_{3}$-continuous.

By Theorem 3 the identity map $\mathscr{M}_{\mathfrak{I}} \rightarrow \mathscr{M}$ is uniformly $\Phi_{3}$-continuous and so,

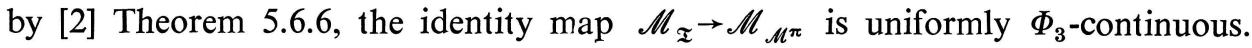
By the above identifications the identity map $\left(\mathscr{C}^{\prime}\right)_{\mathfrak{I}} \rightarrow\left(\mathscr{C}^{\prime}\right)_{\mathscr{C}}$ " is uniformly $\Phi_{3}$-continuous. 
Assume now $Y$ is $\sigma$-Stonian. Then every set of $\mathfrak{I}$ is a closed open set of $X$ and therefore the identity map $\left(\mathscr{C}^{\prime}\right)_{\mathscr{C}} \rightarrow\left(\mathscr{C}^{\prime}\right)_{\mathfrak{x}}$ is uniformly continuous. Hence the identity map $\left(\mathscr{C}^{\prime}\right)_{\mathscr{C}} \rightarrow\left(\mathscr{C}^{\prime}\right)_{\mathscr{\mathscr { C }}}$ is uniformly $\Phi_{3}$-continuous.

Remark. If $X$ if $\sigma$-Stonian (or, equivalently, $\mathscr{C}$ is order $\sigma$-complete ([2] Lemma 5.9.15 a $\Leftrightarrow$ b)), then (by taking $Y=X$ ) the identity map $\left(\mathscr{C}^{\prime}\right)_{\mathscr{C}} \rightarrow\left(\mathscr{C}^{\prime}\right)_{\mathscr{G}}$ is uniformly $\Phi_{3}$-continuous.

Theorem 7. Every $\Phi_{3}$-set of $\left(\mathscr{C}^{\prime}\right)_{\mathfrak{I}}$ and every $\Phi_{4}$-set of $\left(\mathscr{C}^{\prime}\right)_{\mathfrak{R}}$ is equicontinuous.

Let $\mathscr{N}$ be a $\Phi_{4}$-set of $\left(\mathscr{C}^{\prime}\right)_{\mathfrak{R}}$. Since $\left(\mathscr{C}^{\prime}\right)_{\mathfrak{R}}=\mathscr{M}$, we deduce by [2] Theorem $4.2 .16 \mathrm{c}$, that there exists an increasing sequence $\left(K_{n}\right)_{n \in N}$ in $\mathfrak{R}$ such that

$$
|\mu|\left(X \backslash K_{n}\right)<\frac{1}{4^{n}}
$$

for every $\mu \in \mathscr{N}$ and $n \in N$. We set $K_{-1}:=\emptyset$,

and

$$
g: X \rightarrow \boldsymbol{R}_{+}, t \mapsto\left\{\begin{array}{cll}
\frac{1}{2^{n-1}} & \text { if } & t \in K_{n} \backslash K_{n-1} \quad(n \in N), \\
0 & \text { if } & t \in X \backslash \bigcup_{n \in N} K_{n},
\end{array}\right.
$$

$$
\mathscr{U}:=\{f \in \mathscr{C}|| f g \mid \leqq 1\} .
$$

Then $\mathscr{U}$ is a 0 -neighbourhood in $\mathscr{C}$. Let $\mu \in \mathscr{N}$ and $f \in \mathscr{U}$. We have

$$
\left|\int f d \mu\right| \leqq \sum_{n \in N} \int_{K_{n} \backslash K_{n-1}}|f| d|\mu| \leqq \sum_{n \in N} \frac{2^{n-1}}{4^{n}}=1 .
$$

Hence $\mathscr{N}$ is equicontinuous.

Assume now $\mathscr{N}$ is a $\Phi_{3}$-set of $\left(\mathscr{C}^{\prime}\right)_{\mathfrak{x}}$. By Theorem 3 and [2] Theorem 1.8.4 a $\Rightarrow \mathrm{h}, \mathscr{N}$ is a $\Phi_{3}$-set of $\left(\mathscr{C}^{\prime}\right)_{\mathfrak{R}}$ and so, by the above considerations, $\mathscr{N}$ is equicontinuous.

Corollary 8. Every boundedly weakly compact continuous linear map $u: \mathscr{C} \rightarrow E$ with respect to the Mackey topology of $\mathscr{C}$ is continuous with respect to the strict topology of $\mathscr{C}$.

Le $A^{\prime}$ be an equicontinuous set of $E^{\prime}$. Since $u$ is boundedly weakly compact, $u^{\prime \prime}\left(\mathscr{C}^{\prime \prime}\right) \subset E$ and so $u^{\prime}\left(A^{\prime}\right)$ is a relatively compact set of $\left(\mathscr{C}^{\prime}\right)_{\mathscr{C}^{\prime \prime}}$. By Theorem 7, $u^{\prime}\left(A^{\prime}\right)$ is equicontinuous; hence $u$ is continuous with respect to the strict topology of $\mathscr{C}$.

Remark. The following example ${ }^{3}$ ) will show that not every circled convex compact $\Phi_{1}$-set of $\left(\mathscr{C}^{\prime}\right)_{\mathscr{C}}$ is equicontinuous, even if $X$ is locally compact and normal.

3) This example appears in [3] Theorem 5 . 
In particular, the strict topology on $\mathscr{C}$ may be strictly coarser than its Mackey topology. Let $\omega_{1}$ be the first uncountable ordinal number, $X$ be the set $\omega_{1}$ endowed with the usual locally compact topology

$$
\left\{V \subset \omega_{1} \mid \xi \in V \Rightarrow \exists \eta<\xi, \quad\left\{\zeta \in \omega_{1} \mid \eta<\zeta \leqq \xi\right\} \subset V\right\},
$$

and for every $\xi \in \omega_{1}$ let $\delta_{\xi}$ be the Dirac measure on $X$ at $\xi$. Then $X$ is locally compact and normal and the circled convex closed hull of

$$
\left\{\delta_{\xi}-\delta_{\xi+1} \mid \xi \in \omega_{1}\right\}
$$

is a compact $\Phi_{1}$-set of $\left(\mathscr{C}^{\prime}\right)_{\mathscr{C}}$, which is not equicontinuous.

Corollary 9. The set $\left\{x^{\prime} \circ \mu \mid x^{\prime} \in A^{\prime}, \mu \in \mathscr{N}\right\}$ is an equicontinuous set of $\mathscr{C}^{\prime}$ for every equicontinuous set $A^{\prime}$ of $E^{\prime}$ and for every $\Phi_{4}$-set $\mathscr{N}$ of $\mathscr{M}(E)$.

We show first that the map

$$
A_{E}^{\prime} \times \mathscr{M}(E) \rightarrow \mathscr{M}, \quad\left(x^{\prime}, \mu\right) \mapsto x^{\prime} \circ \mu
$$

is continuous. Let $\left(x_{0}^{\prime}, \mu_{0}\right) \in A^{\prime} \times \mathscr{M}(E)$ and let $A \in \Re$ and $\varepsilon>0$. There exist a 0 -neighbourhood $U$ in $E$ such that $\left|x^{\prime}(x)\right|<\varepsilon / 2$ for every $\left(x^{\prime}, x\right) \in A^{\prime} \times U$ and a neighbourhood $V$ of $x_{0}^{\prime}$ in $A_{E}^{\prime}$ such that

$$
\left|x^{\prime}\left(\mu_{0}(A)\right)-x_{0}^{\prime}\left(\mu_{0}(A)\right)\right|<\frac{\varepsilon}{2}
$$

for every $x^{\prime} \in V$. Further, there exists a neighbourhood $\mathscr{W}$ of $\mu_{0}$ in $\mathscr{M}(E)$ such that

for every $\mu \in \mathscr{W}$. We get

$$
\mu(A)-\mu_{0}(A) \in U
$$

$$
\begin{gathered}
\left|x^{\prime} \circ \mu(A)-x_{0}^{\prime} \circ \mu_{0}(A)\right| \leqq\left|x^{\prime}\left(\mu(A)-\mu_{0}(A)\right)\right|+\left|x^{\prime}\left(\mu_{0}(A)\right)-x_{0}^{\prime}\left(\mu_{0}(A)\right)\right| \\
<\frac{\varepsilon}{2}+\frac{\varepsilon}{2}=\varepsilon
\end{gathered}
$$

for every $\left(x^{\prime}, \mu\right) \in V \times \mathscr{W}$. Hence the map

is continuous.

$$
A_{E}^{\prime} \times \mathscr{M}(E) \rightarrow \mathscr{M}, \quad\left(x^{\prime}, \mu\right) \mapsto x^{\prime} \circ \mu
$$

In order to prove the assertion of the corollary we may assume $E$ complete. Then $\mathscr{N}$ is a relatively compact set of $\mathscr{M}(E)$ ([2] Theorem 4.2.16 a)) and by the above considerations $\left\{x^{\prime} \circ \mu \mid x^{\prime} \in A^{\prime}, \mu \in \mathscr{N}\right\}$ is a relatively compact set of $\mathscr{M}$. By Theorem 7 this set is equicontinuous.

Proposition 10. Let $E$ be quasicomplete and $\mathscr{L}_{0}(\mathscr{C}, E)$ be the vector space of boundedly weakly compact continuous linear maps of $\mathscr{C}$ into $E$. We denote by $\bar{\mu}$ the map

for every $\mu \in \mathscr{M}(E)$. Then

$$
\mathscr{C} \rightarrow E, \quad f \mapsto \int f d \mu
$$


a) $\{\bar{\mu} \mid \mu \in \mathscr{N}\}$ is an equicontinuous set of $\mathscr{L}_{0}(\mathscr{C}, E)$ for every $\Phi_{4}$-set $\mathscr{N}$ of $\mathscr{M}(E)$ :

b) the map

$$
\mathscr{M}(E) \rightarrow \mathscr{L}_{0}(\mathscr{C}, E), \quad \mu \mapsto \bar{\mu}
$$

is an isomorphism of vector spaces;

c) for every $\mu \in \mathscr{M}(E)$ the maps

$$
\begin{aligned}
& E^{\prime} \rightarrow \mathscr{C}^{\prime}, \quad x^{\prime} \mapsto x^{\prime} \circ \mu \\
& \mathscr{C}^{\prime \prime} \rightarrow E, \quad \xi \mapsto \int \xi d \mu
\end{aligned}
$$

are the adjoint and the biadjoint of $\bar{\mu}$ and the map

$$
(\mathscr{C})_{\mathscr{C}^{\prime}} \rightarrow E, \quad f \mapsto \int f d \mu
$$

is uniformly $\Phi_{4}$-continuous;

d) every $\Phi_{3}$-set of $\mathscr{L}_{0}(\mathscr{C}, E)_{\mathfrak{T}}$ is equicontinuous; in particular, if $Y$ is $\sigma$-Stonian, then every $\Phi_{3}$-set of $\mathscr{L}_{0}(\mathscr{C}, E)_{\mathscr{C}}$ is equicontinuous.

Let $\mu \in \mathscr{M}(E)$. By Proposition 5 a), $\mathscr{C} \subset \mathscr{L}^{1}(\mu)$ and by [1] Theorem 4.2.11, $\int f d \mu \in E$ for every $f \in \mathscr{C}$.

a) Let $A^{\prime}$ be an equicontinuous set of $E^{\prime}$. By Corollary $9,\left\{x^{\prime} \circ \mu \mid x^{\prime} \in A^{\prime}, \mu \in \mathscr{N}\right\}$ is an equicontinuous set of $\mathscr{C}^{\prime}$. Since $A^{\prime}$ is arbitrary, $\{\bar{\mu} \mid \mu \in \mathscr{N}\}$ is an equicontinuous set of linear maps of $\mathscr{C}$ into $E$. By [1] Theorem 4.2.11 this set is contained in $\mathscr{L}_{0}(\mathscr{C}, E)$.

b) It is obvious that the map

$$
\mathscr{M}(E) \rightarrow \mathscr{L}_{0}(\mathscr{C}, E), \mu \mapsto \bar{\mu}
$$

is injective and linear. By [1] Proposition 4.3.9 a) this map is surjective.

c) Let $x^{\prime} \in E^{\prime}$. Then

$$
\left(\bar{\mu}^{\prime}\left(x^{\prime}\right)\right)(f)=x^{\prime}(\bar{\mu}(f))=x^{\prime}\left(\int f d \mu\right)=\int f d\left(x^{\prime} \circ \mu\right)=\left(x^{\prime} \circ \mu\right)(f)
$$

for every $f \in \mathscr{C}$ and so $\bar{\mu}^{\prime}\left(x^{\prime}\right)=x^{\prime} \circ \mu$. Hence

is the adjoint map of $\bar{\mu}$.

$$
E^{\prime} \rightarrow \mathscr{C}^{\prime}, \quad x^{\prime} \mapsto x^{\prime} \circ \mu
$$

Let $\xi \in \mathscr{C}^{\prime \prime}=\mathscr{M}^{\pi}$. Then

$$
\left(\bar{\mu}^{\prime \prime}(\xi)\right)\left(x^{\prime}\right)=\xi\left(\bar{\mu}^{\prime}\left(x^{\prime}\right)\right)=\xi\left(x^{\prime} \circ \mu\right)=\int \xi d\left(x^{\prime} \circ \mu\right)=\left(\int \xi d \mu\right)\left(x^{\prime}\right)
$$

for every $x^{\prime} \in E^{\prime}$ and so $\bar{\mu}^{\prime \prime}(\xi)=\int \xi d \mu$. Hence ([1] Theorem 4.2.11)

is the biadjoint map of $\bar{\mu}$.

$$
\mathscr{C}^{\prime \prime} \rightarrow E, \quad \xi \mapsto \int \xi d \mu
$$

By [2] Corollary 5.8.26, $\mathscr{C}$ endowed with the order relation induced by $\boldsymbol{R}^{X}$ is an $M$-space. By [2] Corollary 5.7.7 the map

$$
(\mathscr{C})_{\mathscr{C}^{\prime}} \rightarrow E, \quad f \mapsto \int f d \mu
$$

is uniformly $\Phi_{4}$-continuous. 
d) Let $\mathscr{N}$ be a $\Phi_{3}$-set of $\mathscr{L}_{0}(\mathscr{C}, E)_{\mathfrak{z}}$. By b) we may consider $\mathscr{N}$ to be a $\Phi_{3}$-set of $\mathscr{M}(E)_{\mathfrak{T}}$ and so, by Corollary 4 and [2] Theorem $1.8 .4 \mathrm{a} \Rightarrow \mathrm{h}$, it is a $\Phi_{3}$-set of $\mathscr{M}(E)$. Let $A^{\prime}$ be an equicontinuous set of $E^{\prime}$. By Corollary 9 there exists a 0 -neighbourhood $\mathscr{U}$ in $\mathscr{C}$ such that

$$
\left|x^{\prime}\left(\int f d \mu\right)\right|=\left|\int f d\left(x^{\prime} \circ \mu\right)\right| \leqq 1
$$

for every $f \in \mathscr{U}$ and every $\left(x^{\prime}, \mu\right) \in A^{\prime} \times \mathscr{N}$. Hence $\mathscr{N}$ is an equicontinuous set of $\mathscr{L}_{0}(\mathscr{C}, E)$.

If $Y$ is $\sigma$-Stonian, then every set of $\mathfrak{I}$ is a closed open set of $X$, so every $\Phi_{3}$-set of $\mathscr{L}_{0}(\mathscr{C}, E)_{\mathscr{C}}$ is a $\Phi_{3}$-set of $\mathscr{L}_{0}(\mathscr{C}, E)_{\mathfrak{I}}$ and it is equicontinuous by the above considerations.

Remark. The assertion b) was proved by A. Grothendieck ([6] Proposition 14) for $X$ compact.

Proposition 11. Let $A^{\prime \prime}$ be a subset of $E^{\prime \prime}$ such that the identity map $E_{A^{\prime \prime}}^{\prime} \rightarrow E_{E^{\prime \prime}}^{\prime}$ is sequentially continuous, $F$ be a $G$-space and $u: E \rightarrow F$ be a continuous linear map such that $u^{\prime \prime}\left(A^{\prime \prime}\right) \subset F$. If $E$ possesses the strong DP-property, we have:

a) the map $E_{E^{\prime}} \rightarrow F$ defined by $u$ is uniformly $\Phi_{4}$-continuous;

b) if in addition $E$ possesses the D-property and $F$ is quasicomplete, then $u$ is boundedly weakly compact.

a) Let $\mathfrak{U}^{\prime}$ be the set of $\Phi_{4}$-sets of $E_{E^{\prime \prime}}^{\prime}$ and $\mathfrak{B}^{\prime}$ be the set of $\Phi_{1}$-sets of $F_{F}^{\prime}$. Let $B^{\prime} \in \mathfrak{B}^{\prime}$. Since $u^{\prime \prime}\left(A^{\prime \prime}\right) \subset F$, the map $F_{F}^{\prime} \rightarrow E_{A^{\prime \prime}}^{\prime}$ defined by $u^{\prime}$ is continuous and so $u^{\prime}\left(B^{\prime}\right)$ is a $\Phi_{1}$-set of $E_{A^{\prime \prime}}^{\prime}$. The map $E_{A^{\prime \prime}} \rightarrow E_{E^{\prime \prime}}^{\prime}$ being sequentially continuous, $u^{\prime}\left(B^{\prime}\right)$ is a $\Phi_{1}$-set of $E_{E^{\prime \prime}}^{\prime}$ ([2] Proposition 1.5.4. a $\left.\Leftrightarrow \mathrm{c}\right)$ and so it belongs to $\mathfrak{A}^{\prime}$. Hence $u^{\prime}\left(\mathfrak{B}^{\prime}\right) \subset \mathfrak{U}^{\prime}$ and the map $E_{\mathfrak{Q}^{\prime}} \rightarrow F_{\mathfrak{B}^{\prime}}$ defined by $u$ is continuous. Since $E$ possesses the strong DP-property, the identity map $E_{E^{\prime}} \rightarrow E_{\mathfrak{H}^{\prime}}$ is uniformly $\Phi_{4}$-continuous. Since $F$ is a $G$-space, the identity map $F_{\mathfrak{B}^{\prime}} \rightarrow F$ is uniformly $\Phi_{4}$-continuous. Putting together the above results we deduce by [2] Corollary 1.8.5 that the map $E_{E^{\prime}} \rightarrow F$ defined by $u$ is uniformly $\Phi_{4}$-continuous.

b) Let $\left(x_{n}\right)_{n \in N}$ be a weak Cauchy sequence in $E$. By a), $\left(u\left(x_{n}\right)\right)_{n \in N}$ is a Cauchy sequence and so a convergent sequence in $F$. Since $E$ possesses the D-property, $u$ is boundedly weakly compact.

Theorem 12. Let $E$ be $a G$-space and $u: \mathscr{C} \rightarrow E$ be a continuous linear map such that (with the usual identifications) $u^{\prime \prime}\left(1_{T}^{X}\right) \in E$ for every $T \in \mathfrak{I}$ (this condition is automatically fulfilled if $Y$ is $\sigma$-Stonian). We have:

a) the map $\mathscr{C}_{\mathscr{G}^{\prime}} \rightarrow E$ defined by $u$ is uniformly $\Phi_{4}$-continuous;

b) if $E$ quasicomplete, then $u$ is boundedly weakly compact and there exists a unique $\mu \in \mathscr{M}(E)$ such that $\int \xi d \mu \in E$ for every $\xi \in \mathscr{M}^{\pi}$,

for every $f \in \mathscr{C}$,

$$
u(f)=\int f d \mu
$$

$$
u^{\prime}\left(x^{\prime}\right)=x^{\prime} \circ \mu
$$


for every $x^{\prime} \in E^{\prime}$, and

for every $\xi \in \mathscr{C}^{\prime \prime}$.

$$
u^{\prime \prime}(\xi)=\int \xi d \mu
$$

By Theorem 6 the identity map $\left(\mathscr{C}^{\prime}\right)_{\mathfrak{T}} \rightarrow\left(\mathscr{C}^{\prime}\right)_{\mathscr{C}^{\prime \prime}}$ is uniformly $\Phi_{3}$-continuous. By [2] Corollary 5.8.26, $\mathscr{C}$ endowed with the order relation induced by $\boldsymbol{R}^{X}$ is an $M$-space and so by [2] Corollary 5.7.9 and Theorem 5.8.9 c) it possesses the strong DP-property and the D-property. Hence by Proposition 11 the map $\mathscr{C}_{\mathscr{C}^{\prime}} \rightarrow E$ defined by $u$ is uniformly $\Phi_{4}$-continuous and $u$ is boundedly weakly compact if $E$ is quasicomplete. The other assertions follow from Proposition 10.

If $Y$ is $\sigma$-Stonian, then every set of $\mathfrak{I}$ is a closed open set of $X$ and so $1_{T}^{X} \in \mathscr{C}$ and $u^{\prime \prime}\left(1_{T}^{X}\right)=u\left(1_{T}^{X}\right) \in E$ for every $T \in \mathfrak{I}$.

Corollary 13. If $Y$ is $\sigma$-Stonian and $\mathscr{C}$ is a G-space, then every compact set of $X$ is finite and $\mathscr{C}$ is semi-separable.

Let $K$ be a compact set of $X$ and $\mathscr{F}$ be the Banach space of continuous real functions on $K$. By Theorem 12 a) the identity map $\mathscr{C}_{\mathscr{C}^{\prime}} \rightarrow \mathscr{C}$ is uniformly $\Phi_{4}$-continuous and so the map

$$
\mathscr{C}_{\mathscr{C}^{\prime}} \rightarrow \mathscr{F}, \quad f \mapsto f \mid K
$$

is also uniformly $\Phi_{4}$-continuous. Let $\left(f_{n}\right)_{n \in N}$ be a weak Cauchy sequence in $\mathscr{C}$. Then, by the above result, $\left(f_{n} \mid K\right)_{n \in N}$ is a Cauchy sequence and so a convergent sequence. By [2] Corollary 5.8.26 and Theorem 5.8.9 a) the map

$$
\mathscr{C} \rightarrow \mathscr{F}, \quad f \mapsto f \mid K
$$

is boundedly weakly compact; hence the balls of $\mathscr{F}$ are weakly compact. We deduce $K$ is finite.

Let $g$ be a positive real function on $X$ such that $\{g \geqq \varepsilon\}$ is relatively compact for every $\varepsilon>0$. Then $\{g>0\}$ is countable. We denote by $\mathscr{H}$ the set of real functions $h$ on $\{g>0\}$ such that $\{h \neq 0\}$ is finite and

$$
h(\{g>0\}) \subset Q \cap[-1,1] .
$$

Then $\mathscr{H}$ is also countable and for every $h \in \mathscr{H}$ there exists an $h^{\prime} \in \mathscr{C}$ such that $\left|h^{\prime}\right| \leqq 1$ and $h^{\prime}=h$ on $\{h \neq 0\}$. Then

$$
\left\{\alpha h^{\prime} \mid \alpha \in \boldsymbol{Q}, h \in \mathscr{H}\right\}
$$

is countable and for every $f \in \mathscr{C}$ and every $\varepsilon>0$ there exist $\alpha \in \boldsymbol{Q}$ and $h \in \mathscr{H}$ such that

$$
\sup _{x \in X}\left|\left(\alpha h^{\prime}(x)-f(x)\right) g(x)\right|<\varepsilon
$$

Hence $\mathscr{C}$ is semi-separable. 
Remark. Let $\mathfrak{F}$ be a filter on $\boldsymbol{N}$ finer than the section filter of $\boldsymbol{N}$ and $x$ be a point not belonging to $N$. We set $X:=N \cup\{x\}$ and endow $X$ with the topology

$$
\{V \subset X \mid x \in V \Rightarrow V \cap N \in F\}
$$

Then $X$ is a nondiscrete paracompact space. If $\mathfrak{F}$ is an ultrafilter, then $X$ is $\sigma$-Stonian and its compact sets are finite. If there exist two different ultrafilters $\mathfrak{F}^{\prime}, \mathfrak{F}^{\prime \prime}$ on $N$ such that $\mathfrak{F}=\mathfrak{F}^{\prime} \cap \mathfrak{F}^{\prime \prime}$, then the compact sets of $X$ are finite but $X$ is not $\sigma$-Stonian.

Proposition 14. Let $Z$ be a topological space such that the neighbourhood filter of every point of $Z$ belongs to $\hat{\Phi}_{1}(Z)$, let $\left(\mu_{z}\right)_{z \in Z}$ be a family in $\mathscr{M}(E)$ such that the map

$$
Z \rightarrow E, \quad z \mapsto \mu_{z}(T)
$$

is continuous for every $T \in \mathfrak{T}$, and let $\xi \in \mathscr{M}^{\pi}$ be such that $\int \xi d \mu_{z} \in E$ for every $z \in Z$. Then the map

is continuous.

$$
Z \rightarrow E, \quad z \mapsto \int \xi d \mu_{z}
$$

We may assume $E$ complete. By Corollary 4 the identity map $\mathscr{M}(E)_{\mathfrak{I}} \rightarrow \mathscr{M}(E)_{\mathcal{M}^{\pi}}$ is uniformly $\Phi_{3}$-continuous and so ([2] Proposition 1.8.3) $\Phi_{1}$-continuous. By the hypothesis the map

$$
Z \rightarrow \mathscr{M}(E)_{\mathfrak{T}}, \quad z \mapsto \mu_{z}
$$

is continuous, and so the map

$$
Z \rightarrow \mathscr{M}(E)_{\mathscr{M}^{\pi}}, \quad z \mapsto \mu_{z}
$$

is $\Phi_{1}$-continuous. Since the neighbourhood filter of every point of $Z$ belongs to $\widehat{\Phi}_{1}(Z)$, this map is continuous ([2] Proposition 1.3.6). This is exactly the assertion that the map

$$
Z \rightarrow E, \quad z \mapsto \int \xi d \mu_{z}
$$

is continuous for every $\xi \in \mathscr{M}^{\pi}$.

Theorem 15. Let $Z$ be a Hausdorff topological space such that the neighbourhood filter of every point of $Z$ possesses a countable base, $\mathscr{F}$ be the vector space of continuous maps of $Z$ into $E$ endowed with the topology of compact convergence and $\left(\mu_{z}\right)_{z \in Z}$ be a family in $\mathscr{M}(E)$ such that the map

$$
Z \rightarrow E, \quad z \mapsto \mu_{z}(T)
$$

is continuous for every $T \in \mathfrak{I}$. If $E$ is quasicomplete, we have:

a) $\mathscr{F}$ is quasicomplete;

b) there exists a unique $\mu \in \mathscr{M}(\mathscr{F})$ such that $\int \xi d \mu \in \mathscr{F}, \int \xi d \mu_{z} \in E$ and

for every $\xi \in \mathscr{M}^{\pi}$ and $z \in Z$;

$$
\left(\int \xi d \mu\right)(z)=\int \xi d \mu_{z}
$$


c) the map

$$
\mathscr{C} \rightarrow \mathscr{F}, \quad f \mapsto \int f d \mu
$$

is continuous and boundedly weakly compact,

$$
\mathscr{M}^{\pi} \rightarrow \mathscr{F}, \quad \xi \mapsto \int \xi d \mu
$$

is its biadjoint map and the map

is uniformly $\Phi_{4}$-continuous.

$$
\mathscr{C}_{\mathscr{C},} \rightarrow \mathscr{F}, f \mapsto \int f d \mu
$$

a) For every $z \in Z$ let $\psi_{z}$ be the map

$$
\mathscr{F} \rightarrow E, \quad f \mapsto f(z)
$$

and let $\mathfrak{F}$ be a Cauchy filter on $\mathscr{F}$ possessing a bounded set of $\mathscr{F}$. Then $\psi_{z}(\mathfrak{F})$ converges for every $z \in Z$. We set

$$
f: Z \rightarrow E, \quad z \mapsto \lim \psi_{z}(\mathfrak{F}) .
$$

The restriction of $f$ to every compact set of $Z$ is continuous. Since the neighbourhood filter of every point of $Z$ possesses a countable base, $f$ is continuous. It is easy to see that $\mathfrak{F}$ converges to $f$ in $\mathscr{F}$. Hence $\mathscr{F}$ is quasicompact.

b) By a) and [1] Proposition 4.2.11, $\int \xi d \mu \in \mathscr{F}$ and $\int \xi d \mu_{z} \in E$ for every $\xi \in \mathscr{M}^{\pi}$, $\mu \in \mathscr{M}(\mathscr{F})$, and $z \in Z$. By [2] Proposition 1.5.31 the neighbourhood filter of every point of $Z$ belongs to $\Phi_{1}(Z)$ and so, by Proposition 14, the map

$$
Z \rightarrow E, \quad z \mapsto \int \xi d \mu_{z}
$$

is continuous for every $\xi \in \mathscr{M}^{\pi}$. We set

for every $A \in \mathfrak{R}$ and

$$
\mu(A): Z \rightarrow E, \quad z \mapsto \mu_{z}(A)
$$

$$
\mu: \mathfrak{R} \rightarrow \mathscr{F}, \quad A \mapsto \mu(A) .
$$

By [2] Theorem 4.6.3 b) $\mu \in \mathscr{M}(\mathfrak{R}, \mathscr{F} ; \mathfrak{R})$, and by [2] Proposition 5.6.3

$$
\mathscr{M}(\mathfrak{R}, \mathscr{F} ; \Re)=\mathscr{M}(\mathscr{F}) .
$$

Let $z \in Z$ and $x^{\prime} \in E^{\prime}$. We set

$$
\varphi: \mathscr{F} \rightarrow \boldsymbol{R}, \quad f \mapsto x^{\prime}(f(z)) .
$$

The function $\varphi$ is a continuous linear form and

$$
\varphi \circ \mu(A)=x^{\prime}((\mu(A))(z))=x^{\prime}\left(\mu_{z}(A)\right)=x^{\prime} \circ \mu_{z}(A)
$$

for every $A \in \Re$ and so $\varphi \circ \mu=x^{\prime} \circ \mu_{z}$. Let $\xi \in \mathscr{M}^{\pi}$. We have

$$
x^{\prime}\left(\left(\int \xi d \mu\right)(z)\right)=\varphi\left(\int \xi d \mu\right)=\int \xi d(\varphi \circ \mu)=\int \xi d\left(x^{\prime} \circ \mu_{z}\right)=x^{\prime}\left(\int \xi d \mu_{z}\right) .
$$


Since $x^{\prime}$ is arbitrary, we deduce

The unicity of $\mu$ is trivial.

$$
\left(\int \xi d \mu\right)(z)=\int \xi d \mu_{z} .
$$

c) follows from a) and Proposition 10.

Theorem 16. Let $E$ be semi-separable, $Z$ be a locally metrizable topological space, $\mathscr{F}$ be the vector space of continuous maps of $Z$ into $E$ endowed with the topology of compact convergence and $u: \mathscr{C} \rightarrow \mathscr{F}$ a continuous map such that (with the usual identifications) $u^{\prime \prime}\left(1_{T}^{X}\right) \in \mathscr{F}$ for every $T \in \mathfrak{T}$. We have:

a) the map $\mathscr{C}_{\mathscr{G}^{\prime}} \rightarrow \mathscr{F}$ defined by $u$ is uniformly $\Phi_{4}$-continuous;

b) If $E$ is quasicomplete, then $u$ is boundedly weakly compact and there exist uniquely a $\mu \in \mathscr{M}(\mathscr{F})$ and a family $\left(\mu_{z}\right)_{z \in Z}$ in $\mathscr{M}(E)$ such that

$$
\begin{aligned}
& u(f)=\int f d \mu, \quad(u(f))(z)=\int f d \mu_{z}, \\
& u^{\prime \prime}(\xi)=\int \xi d \mu, \quad\left(u^{\prime \prime}(\xi)\right)(z)=\int \xi d \mu_{z}
\end{aligned}
$$

for every $f \in \mathscr{C}, \xi \in \mathscr{C}^{\prime \prime}$ and $z \in Z$.

By [2] Proposition 5.9.30, $\mathscr{F}$ is a $G$-space and, by Theorem $15 \mathrm{a}$ ), it is quasicomplete if $E$ is quasicomplete. By Theorem 12 the map $\mathscr{C}_{\mathscr{C}^{\prime}} \rightarrow \mathscr{F}$ defined by $u$ is uniformly $\Phi_{4}$-continuous, and if $E$ is quasicomplete, then there exists a unique $\mu \in \mathscr{M}(\mathscr{F})$ such that

and

$$
u(f)=\int f d \mu
$$

$$
u^{\prime \prime}(\xi)=\int \xi d \mu
$$

for every $f \in \mathscr{C}$ and $\xi \in \mathscr{C}^{\prime \prime}$. Let $z \in Z$ and let $v$ be the map

$$
\mathscr{C} \rightarrow E, \quad f \mapsto(u(f))(z) .
$$

Then $v$ is a continuous map such that $v^{\prime \prime}\left(1_{T}^{X}\right) \in E$ for every $T \in \mathfrak{T}$. By the above considerations there exists a unique $\mu_{z} \in \mathscr{M}(E)$ such that

for every $f \in \mathscr{C}$. We have

$$
v(f)=\int f d \mu_{z}
$$

$$
\int f d \mu_{z}=v(f)=(u(f))(z)=\left(\int f d \mu\right)(z)
$$

for every $f \in \mathscr{C}$ and so

for every $A \in \Re$ and

$$
\mu_{z}(A)=(\mu(A))(z)
$$

for every $\xi \in \mathscr{C}^{\prime \prime}$.

$$
\int \xi d \mu_{z}=\left(\int \xi d \mu\right)(z)=\left(u^{\prime \prime}(\xi)\right)(z)
$$




\section{References}

[1] Constantinescu, C.: Duality in measure theory. - Lecture Notes in Mathematics 796. SpringerVerlag, Berlin-Heidelberg-New York, 1980.

[2] Constantinescu, C.: Spaces of measures. - de Gruyter Studies in Mathematics 4. Walter de de Gruyter, Berlin-New York, 1984.

[3] Conway, J.B.: The strict topology and compactness in the space of measures. - Bull. Amer. Math. Soc. 72, 1966, 75-78.

[4] GÄNSSLER, P.: A convergence theorem for measures in regular Hausdorff spaces. - Math. Scand. 29, 1971, 237-244.

[5] GiLEs, R.: A generalization of the strict topology. - Trans. Amer. Math. Soc. 161, 1971, 467-474.

[6] Grothendieck, A.: Sur les applications linéaires faiblement compactes d'espaces du type $C(K)$. - Canadian J. Math. 5, 1953, 129-173.

[7] Khurana, S. S.: Convergent sequences of regular measures. - Bull. Acad. Polon. Sci. Sér. Sci. Math. Astronom. Phys. 24, 1976, 37-42.

[8] Semadeni, Z.: On weak convergence of measures and $\sigma$-complete Boolean algebras. - Colloq. Math. 12, 1964, 229-233.

Eidgenössische Technische Hochschule

Mathematisches Seminar

CH-8092 Zürich

Switzerland

Received 14 November 1983 